\title{
Rotavirus: Preventive Vaccines and a Rare Side Effect
}

\author{
Harini Rajagopal ${ }^{1}$, Nithya $\mathrm{T}^{2}$, Hemlin Thomas $^{3}$, Varghese $\mathrm{P} \mathrm{R}^{4}$ and Aparna Namboodirippad ${ }^{5 *}$ \\ 1,2, $3 \& 5$ Department of Paediatrics, ${ }^{4}$ Jubilee Centre for Medical Research, \\ Jubilee Mission Medical College \& research Institute, Thrissur, Kerala, India
}

\begin{abstract}
Rotaviruses are the most common cause of diarrhoeal disease among infants. Till now 108 countries have introduced rotavirus vaccines to prevent severe diarrhea. The Global Rotavirus Surveillance Network (GRSN) was established by WHO in 2008. Some studies reported that rotavirus vaccination can lead to a small increase in the risk of intussusception, a type of bowel blockage. This is a rare side effect. Intussusception, death of tissue of the intestinal wall due to lack of blood supply may cause life-threatening complications if left untreated. In infants idiopathic ileocolic intussusception is the most common form typically managed with operative or non operative reduction like hydrostatic or pneumatic enemas. Whereas, in adults and older children, intussusception is not very common and occurs more often in the colon of small intestine, pathogenesis is lead point in most of the symptomatic cases. Numerous countries have scrupulously noted the occurrence of intussusception post vaccination, and others yet to introduce the vaccine are gathering base line data on the incidence of intussusception which is an important safety issue in determining vaccine policy. However, it should be noted that naturally occurring intussusception, although rare, does occur in the very young. Due to heavy burden of rotavirus, some developing countries have introduced rotavirus vaccines. In India it is now part of the national immunization programme. Hospitalizations and deaths from rotavirus have been averted significantly because vaccination far exceeded the reported cases of intussusception with vaccination. The underlying rationale behind the differing risk of intussusception after rotavirus vaccination in various countries is not yet determined, therefore continuous monitoring is necessary.
\end{abstract}

Keywords:- Infection; Rotavirus; Rotavirus Vaccine; Immunization; Intussusceptions; Side Effects.

\section{INTRODUCTION}

Rotavirus belongs to the family of Reoviridae which is a genus of RNA viruses. Almost every child in the world is infected with rotavirus at least once by five years of age. We can protect babies against rotavirus disease with safe and effective vaccination. Due to heavy burden of rotavirus, 70\% sub-Saharan African countries have introduced rotavirus vaccines. In Asia, only few countries have introduced the vaccine nationally or sub-nationally. Rotavirus vaccines are now introduced in India as a part of immunization regime. Rotavirus is diagnosed with the help of a stool sample in a medical laboratory [1].
Some studies reported that rotavirus vaccination can lead to a small increase in the risk of intussusception, a type of bowel blockage, telescoping of adjacent tubular sections of the intestine. This side effect is very rare. Acute viral gastroenteritis caused by adenovirus, rotavirus, norovirus, and others have been implicated in intussusception cases, with adenovirus detected in the highest proportion. Naturally occurring intussusception is predominantly observed in infants aged 4 to 10 months. This article reviews the present knowledge on rotavirus, its treatment, preventive vaccination and one of the rare side effects of vaccination, the intussusceptions $[2,3]$.

\section{$>$ Symptoms of rotavirus infection}

This occurs very frequently in young children; adults can also get the infection, although it's usually less severe. The symptoms can start within two days after being exposed to the rotavirus. This may range from mild to severe and child-to-child. The most common symptoms include: fever, which usually subsides within the first couple of days, nausea and vomiting which is hallmark of rotavirus disease, abdominal pain, diarrhea (may last between three to eight days), dehydration, can occur quickly, especially in infants. Symptoms of dehydration may include: lethargy or sleepiness, irritability, thirst, pale color to skin or mottling, less elasticity in the skin, eyes appear deeply sunken, baby's fontanelle (or soft spot) may feel sunken, decreased or absent tears, decreased urine output or fewer wet diapers, dry mouth. Even if the symptom disappears the infection might last up to 10 days in the stool [4].

\section{$>$ Rotavirus transmission}

Rotavirus is present in stool, mainly transmitted through hand and mouth contact. More infection spreads during winter and spring months. Infants and children under age three are more prone for rotavirus infection especially kids attending daycare. This is due to not washing hands after using the toilet or changing diapers. It is crucial to disinfect all common surfaces at home frequently, especially if a member of the household having rotavirus touches the surface, the infection can persist for several days on them.

\section{$>$ Rotavirus treatment}

- Mainly supportive treatment.

- Oral rehydration is the corner stone.

- Consume broth-based soups.

- Drink ORS, Pedialyte or other fluids with electrolytes (important for children). 
- BRAT diet stands for bananas, rice, applesauce and toast. Take a diet of bland foods, such as saltines and white toast.

- Avoid fatty or sugary foods as these can worsen diarrhea.

In case of severe dehydration, hospitalization is suggested following administration of intravenous (IV) fluids to help prevention of life-threatening complications.

\section{Rotavirus vaccines}

After it was first introduced in 2006, hospitalizations and deaths from rotavirus have dropped significantly. The vaccine is available in different forms. Commonly given rotavirus vaccine in India is Rotavac (Bharat) $116 \mathrm{E}$ or Rotasure - Abbott (G9, P11). Rotasure is an injectable 0.5 $\mathrm{ml}$ of the ready to use vaccine indicated for prevention. Given to the infants at minimum age of 6 weeks and three doses are completed by 8 months of age. Non replicating rotavirus vaccine (NRRV - P2-VP8* TV) is in pipeline. Developed by PATH (nonprofit global health organization), Trivalent vaccine truncated VP8 subunits of $\mathrm{P}[4], \mathrm{P}[6]$ and $\mathrm{P}[8]$. The administration route is Parenteral Intra Muscular. Some WHO licensed vaccines are listed in table1 [5]. These vaccines are administered by oral route.

\begin{tabular}{|c|c|c|c|c|}
\hline Vaccine & ROTARIX & ROTATEQ & ROTAVAQ & ROTASIIL \\
\hline Country & Belgium & USA & India & India \\
\hline $\begin{array}{c}\text { Composition } \\
\text { and strain }\end{array}$ & $\begin{array}{c}\text { Human Live, } \\
\text { monovalent } \\
\text { attenuated single } \\
\text { strain R1X4414 } \\
\text { ofG1P[8]vaccine } \\
\text { RV1 }\end{array}$ & $\begin{array}{c}\text { Live pentavalent, } \\
\text { human-bovine } \\
\text { reassortant vaccine } \\
\text { Types G1, G2, G3, } \\
\text { G4, and G9. RV5 }\end{array}$ & $\begin{array}{c}\text { Live attenuated, } \\
\text { monovalent vaccine } \\
\text { containing a P[11] and } \\
\text { 116E of human strain } \\
\begin{array}{c}\text { G9P(11) isolated from } \\
\text { an Indian child }\end{array}\end{array}$ & $\begin{array}{c}\text { Lyophilized pentavalent } \\
\text { vaccine containing } \\
\text { human bovine reassortant } \\
\text { strains of rotavirus } \\
\text { serotypes G1, G2, G3, G4 } \\
\text { and G9. }\end{array}$ \\
\hline Presentation & $\begin{array}{c}\text { Liquid and } \\
\text { lymphosized }\end{array}$ & Liquid & Liquid & Lymphosized \\
\hline Dosage & 2 & 3 & 3 & 3 \\
\hline Schedule & 6,16 Weeks & $6,12,20$ Weeks & $6,10,14$ weeks & $6,10,14$ Weeks \\
\hline $\begin{array}{c}\text { Storage } \\
\text { temperature }\end{array}$ & $2-8 \mathrm{C}$ & $2-8$ & $2-8$ & $<25$ \\
\hline Duration & 3 years & 2 years & 6 Months & 36 Months \\
\hline
\end{tabular}

Table 1:- List of WHO approved vaccines

There are no vaccines available for older children and adults. This is the reason health professionals recommend the rotavirus vaccine for the children at younger age. Though able to contain severity of infection, no vaccine is 100 percent effective. Infants with severe combined immunodeficiency or who are already severely ill shouldn't get the vaccine. Most of the babies who get rotavirus vaccine do not have any complications with it. It is very safe and effective at preventing the disease [6].

In some high and middle income countries, rotavirus vaccination has already shown to reduce hospitalizations among children and adults who are too old to be vaccinated, an effect known as herd immunity. Indirect effects of vaccines may result from horizontal transmission of vaccine leading to immune protection in unvaccinated contacts known as herd immunity and reduction in transmission of wild type infection is known as herd protection. Few vaccines like measles or rubella vaccines provide herd protection only, whereas live, oral vaccines such as rotavirus and Oral Poliovirus Vaccine (OPV) can provide both herd protection and herd immunity. The herd protection can be generated by two ways that is through decrease in the severity of infection in a vaccinated individual if they acquire disease and depletion in the number of infected cases with subsequently low probability of susceptible community members coming in contact with an infectious individual [7].

\section{Side effects of vaccine}

This includes diarrhea, fever, fussiness, irritability, otitis media, nasopharyngitis, and bronchospasm, kawasaki and intussusception. Drastic reduction has been seen in many countries in rotavirus-specific and diarrheal illnesses following vaccine introduction [8].

\section{What is intussusception?}

In intussusception, one part of the intestine which is a long tube like structure- most often the small intestine-slides inside an adjacent part, usually in the segment of bowel which is more distal, the intussuscipiens. Since it folds together like a collapsible telescope, hence is sometimes called telescoping [9].

\section{Etiopathogenesis of intussusception}

Intussusceptions can happen in many instances. The normal wave-like motility of the intestine known as peristaltic movement grabs and pulls the lead point and the lining of the intestine into the bowel ahead of it. Only in few cases, the telescoping occurs due an abnormal growth in the intestine, like a polyp or a tumor called as lead point, while mesenteric lymphadenopathy ensuing venous and lymphatic congestion is the most common cause of intussusception.

\section{Naturally occurring intussusception}

The reason behind intussusception has not been found in $47.8 \%$ of the cases, conclusively occurs at age 4 to 10 month. These ideopathic cases believed to arise from hypertrophy of infected lymph nodes including payer's patches.

Documented baseline of intussusception rates differs by population and also attributed to number of factors such as ethnicity, access to health care, cultural or socio-environmental factors. According to a study intussusception incident rates in Japan has shown 185 cases and Vietnam has shown 302 cases per 100,000 infants which is significantly higher. However middle- and high-income countries has shown $30-70$ cases per 100,000 infants. There is a difference in the case fatality ratio of intussusception as well due to accessibility to health care system which varies between 1-9\% in Asian and African countries and less than $1 \%$ in developed countries and [10]. 
Rotavirus vaccine induced intussusception- a rare side effect

Although the role of rotavirus vaccines in inducing intussusception is low, whether the live, attenuated rotavirus vaccines cause this to happen and if at all it is responsible, why it occurs only in some children is not yet identified. The viable mechanisms include a gastrointestinal infection that might cause substantial enlargement of the Peyer's patches in the terminal ileum, which in turn acts as a lead point inducing alterations in the wave like contractions of the intestine that contribute to the risk of intussusception.

\section{$>$ Risk factors for intussusception}

- Age- more often in infants between 6 months and 3 years of age than in adults.

- Sex- predominanly in boys.

- Abnormal intestinal formation at birth. Intestinal malrotation is a condition in which the intestine doesn't develop or rotate correctly, and it increases the risk for intussusception.

- Those with prior history of intussusception has higher chances of recurrence.

- A family history. Someone who has had an intussusception, their siblings are at a much greater risk of the disorder [11]

\section{$>$ Difference between intussusception in adults and children}

Intussusception is more common in children especially $6-18$ months than in adults accounting for $1-5 \%$ of bowel obstruction cases. Out of all the cases, roughly $30 \%$ of children older than two years gets effected. Due to partial obstruction adults often present with non-specific chronic and more variation in symptoms whereas children usually have the classic symptoms. Nearly $90 \%$ of adults with intussusception develop it as a result of some underlying condition. In contrast only $10 \%$ of children have some identifiable cause usually has no known cause.

\section{EXAMINATION OF INTUSSUSCEPTION}

\section{A. Clinical examination}

On examination, in $2 / 3$ rd of children, a sausage shaped mass in the right abdomen, crossing the midline, behind the umblicus in the epigastrium is felt which might be subtle at times. Performed when the child is settled in between episodes. Bowel obstruction indicated in case abdomen distension. Perforation and peritonitis can be present in case of tenderness or guarding. Nappy and perianal region should be inspected. In very rare cases rectal examination is indicated. Hypovolaemic shock can occur in infants.

\section{B. Investigations of intussusception}

$>$ Ultrasound scan: Point of Care Ultrasound can confirm the diagnosis of intussusception. It should not be used to exclude the diagnosis.

- In transverse direction, "target or doughnut" appearance shows hyperechoic central area that is intussusceptum and associated mesenteric fat surrounded by hypoechoic rim that is edematous bowel wall.
- In longitudinal direction "Sandwich, trident or hayfork" appearance shows hyperechoic mesentery and layering of hypoechoic bowel wall.

- In Oblique direction, "pseudokidney" appearance shows hyperechoic mesentery mimicking the renal fat and hypoechoic bowel wall mimicking the renal cortex.

- Doppler maybe helpful in determining viability of the tissue

In adults, useful only when an palpable abdominal mass is present.

Abdominal X-Ray: Abdominal X-Ray should be performed only in case of obstruction or perforation.

- Crescent sign - A soft tissue mass surrounded by a crescent lucency of bowel gas. Lack of faecal material in the large bowel may also be noticed. Signs of small bowel obstruction and pneumoperitoneum indicate bowel perforation.

- Barium enema - In lumen of the intussusceptum and in the intraluminal space, barium is used for both diagnostic and therapeutic purposes gives a "Coiled spring” appearance. Adults usually show normal bowel gas pattern.

\section{$>$ Computerised tomography (CT): Commonly} performed in adults.

- In transverse direction "target" appearance, with layers of fat and bowel wall visible. If enhanced may see mesenteric vessels in the layers and oral contrast in the intraluminal spaces.

- In longitudinal direction, "sausage-shaped" elongated appearance of mass with visible layers can be seen. Pathological lead point may or may not be present.[12]

\section{* Complications of intussusception}

Intussusception if untreated can cut off the blood supply to the affected portion of the intestine. This condition may further lead to a perforation in the intestinal wall, followed by an infection of the lining of the abdominal cavity called peritonitis. Peritonitis is a life-threatening condition that requires immediate medical attention. Signs and symptoms of peritonitis includeabdominal pain, abdominal swelling and fever. Peritonitis may cause the child to go into shock. A child who is in shock may be conscious or unconscious. Signs and symptoms of shock include- Cool, clammy skin that may be pale or gray, a weak and rapid pulse, abnormal breathing that may be either slow and shallow or very rapid, anxiety or agitation and profound listlessness [13].

\section{* Management}

The treatment for hypovolemic shock is with IV administration of normal saline. Analgesia is given ,usually intranasal fentanyl or IV morphine and resuscitating before diagnostic imaging. Children having ileocolic intussusception, undergoing ultrasound or air and hydrostatic enema can decompensate. 


\section{A. Non operative reduction \\ $>$ Hydrostatic Reduction}

The hydrostatic reduction could be performed by the use of saline, barium, or another solution.

\section{Contrast / gas enema/ pneumatic enema}

The enema can be for diagnostic and therapeutical purpose, there is a small risk of bowel perforation and bacteremia during the gas enema. It should not be performed in case of peritonitis, shock, perforation, or an unstable clinical condition.

\section{B. Surgical Reduction}

On the other hand, surgery is indicated in patients who have recurrent intussusception or other than ileocolic intussusception such as jejuna-jejunal, ileo-ileal or jejunal-ileal intussusception as these regions have higher incidence of a pathologic lead point and also hemodynamically unstable or having evidence of perforation or having other complications must also be managed operatively [14].

\section{* Intussusception prior to vaccine implementation and following vaccine administration \\ Studies documented that almost 1 in 20,000 to 1 in} 100,000 US infants who get rotavirus vaccine within a week might develop intussusception. This means that between 40 and 120 US infants might develop intussusception following rotavirus vaccination in United States and other countries. The first rotavirus vaccine, RotaShield, was withdrawn from the US market in 1999 after $~ 30$-fold increase in risk of intussusception during the week following receipt of the first vaccine dose [15].

Rotarix was associated with a small risk of intussusception, approximately 1 in every 51,000 to 68,000 vaccinated infants in Mexico whereas the risk of intussusception post Rotarix were not found in Brazil [16].

In Taiwan mean rate of intussusception hospitalization were significantly lower during the post-vaccine licensure period for the overall group of children aged less than 12 months [17].

In Korea the incidence of intussusception in pre-vaccine period was greater compared to post-vaccine period. A study was conducted in 3 infant age groups 6-14, 15-24, and 25-34 weeks. It was highest in those aged 15-24 and 25-34 weeks and was lowest in 6-14 weeks old. During the pre-vaccine period, the mean rate of incidence of intussusception in infants aged 6-14 weeks old was 90.8 per 100,000 infants, In the post vaccination era, the reduction rate was $35 \%$ in $2009,69 \%$ in 2011 and $55 \%$ in 2015. During the pre-vaccine era, the mean rate of intussusception in the age group 15-24 weeks old was 256.8 per 100,000 infants and 25-34 weeks old, was 315.7 per 100,000 infants. During the post-vaccine period it was decreased by $25-53 \%$ in infants $15-24$ weeks old and 6-32\% in infants $25-34$ weeks old [18].
A study was conducted in four low income Asian countries that is Bangladesh, Nepal, Pakistan and Vietnam to describe the baseline epidemiology of intussusception in young children before implementation of rotavirus vaccine. This was a two year study of children less than 2 years of age. The percentage of cases underwent surgical management was $100 \%$ in Bangladesh, $88 \%$ in Pakistan, $61 \%$ in Nepal, and $1 \%$ in Vietnam. The high proportion of males and median age of cases around 6 months of age found in this regional surveillance network were in accordance with past descriptions of the epidemiology of intussusception [19].

In India analysis using the Self-Controlled Case Series (SCCS) methodology from the five sites in the states where Rotavac was introduced showed no significant risk of intussusception in any of the risk windows 1-7 days or 1-21 days following immunization, after each dose individually or doses 1 and 2 combined or all three doses combined. A total of 133 children aged less than two years with intussusception diagnosis were recruited. The male-female ratio was $2: 1$. The children aged 2-6 months, 7-12 months, 13-18 months and 19-24 months were 46, 51, 24 and 12 respectively. The overall median age was 8 months and some seasonal variation observed with higher number of intussusception cases during March to June months. No cases observed within the risk period after dose 1 . After dose 2, only one case occurred in the 8-21 days window. After dose 3, 1 case in 1-7 and 7 cases in 8-21 window periods were observed. It is to be noted that the median age of third dose was 115 days, which overlaps with the age of natural occurrence of intussusception [20].

In Israel on an average annual incidence of intussusception among Jewish and Arab children below 5 years age was estimated at 36.1 versus 23.2 per 100,000 ; infants below 12 months of age- 128.1 versus 80.1 per 100,000 . Surgical reductions were done in $23.2 \%$ cases. The incidence of intussusception prior to universal rotavirus immunization was documented in northern Israel. Though lower incidence Arab patients underwent surgery more often than Jewish patients due to delayed hospitalization [21].

A report from Germany detected that an increased risk of intussusception was found among infants who received the first dose of rotavirus vaccine, either RotaTeq or Rotarix, between ages 90-179 days, but not in infants less than 89 days of age [22]

The Global Advisory Committee on Vaccine Safety (GACVS) in December 2011 initially reviewed the safety of currently administered rotavirus vaccines. The Committee noted that the benefit of the vaccines was greater than the potential risk, although they may be associated up to 6-fold increased risk of intussusception, both RotaTeq and Rotarix vaccines had a good safety profile [23]. 
In December 2013, GACVS reviewed additional data acquired from Australia and US. It noted that both countries confirmed a risk of intussusception following vaccination, particularly within the first seven days after the first dose, although traceable risk estimates varied across studies. The Committee concluded that the benefits of the vaccine was larger than the small potential risk of intussusception almost 1-2 cases per 100,000 first doses and suggested that given possible population differences in the risk of intussusception, active surveillance should be undertaken in countries where rotavirus vaccines are being introduced to ensure that benefits and risks can continued to be assessed [24].

The GACVS session in December 2017 reviewed recent evidence on the impact of rotavirus vaccine, an updated Cochrane review on rotavirus vaccines and intussusception. SCCS study resulted in an increased risk of intussusception in the weeks after administration of vaccination; however RCTS (randomized control trials) did not indicate any relationship between intussusception and vaccination which is somewhat conflicting in nature [25].

In 2014 African intussusception Surveillance Network that include seven countries using Rotarix that is Ethiopia, Ghana, Kenya, Malawi, United Republic of Tanzania, Zambia and Zimbabwe had seen strong association between oral rotavirus vaccine and intussusception and analysed using a SCCS. No increased risk of intussusception was identified after dose 1 and a small 2-fold risk in the first 7 days but no large scale increased risk was identified from 121 days following the second dose. Post vaccination observation is still conducted [25].

\section{* Steps taken to mitigate intussusception}

Since there is no known cause, there is no way to prevent or avoid intussusception

- It is assumed that a parenteral rotavirus vaccine will not trigger intussusception, although a vaccine that elicits a robust immune response in gut lymphoid tissue could possibly pose a threat, irrespective of the route of administration. Therefore, assessment of intussusception risk with the vaccine will still be needed in future clinical trials [26].

- Vaccination should be provided within the recommended time window in order to keep the risk of intussusception absolute minimum.

\section{CONCLUSION}

The paramount variation in the probable risk of intussusception after rotavirus vaccination in different countries has not been completely elucidated. It may be ascribable to differences in age at which the first dose of vaccination is given; time elapsed from administration of vaccine to development of intussusception; differences in effectiveness of vaccine for example contemporaneous use of inactivated polio vaccine (IPV) and oral poliovirus vaccine (OPV) may reduce both risk of intussusception and effectiveness of the rotavirus vaccine or environmental differences, including circulating pathogens, genetic predisposition dietary pattern of infants, breastfeeding, and accessibility to medical care. Both wild-type and vaccine strain, is associated with the development of intussusception, it could be a reason for this decreasing trend of intussusception. It can be attributed that vaccination could potentially trigger intussusception in a susceptible individual who may be vulnerable to intussusception later in the absence of vaccination. Increased ileum wall thickness and lymph node size were identified in infants with rotavirus infection and healthy controls. How to reduce risk of intussusception induced by rotavirus vaccine is an exigent question. However as the countries are transiting from OPV to IPV, it is considered crucial to evaluate both the effectiveness and risk of intussusception along with that incessant assessment of risk of new vaccines should be done when they are licensed and introduced. Overall, the benefit of rotavirus vaccination in preventing severe diarrhea preponderates the rare adverse effect of vaccination that is intussusception.

\section{REFERENCES}

[1]. Crawford SE, Ramani S, Tate JE, Parashar UD, Svensson L, Hagbom M, Franco MA, Greenberg HB, O'Ryan M, Kang G, Desselberger U. Rotavirus infection. Nature Reviews Disease Primers. 2017 Nov 9;3(1):1-6.

[2]. Jain S, Haydel MJ. Child intussusception 2017.

[3]. Borkar VV, Poddar U, Thakral A, Agarwal J, Srivastava A, Yachha SK, Kumar S. Intussusception in celiac disease: Is it a common feature in children?.Journal of gastroenterology and hepatology. 2018 Feb;33(2):380-4.

[4]. www..mayoclinic.org/diseases-conditions/rotavirus/s ymptoms-causes/syc-20351300

[5]. www.researchgate.net/figure/Rotavirus-vaccines-ava ilable-in-India tbl2 337446241

[6]. Wang CM, Chen SC, Chen KT. Current status of rotavirus vaccines. World Journal of Pediatrics. 2015 Nov 1;11(4):300-8.,

[7]. Parashar UD, Cortese MM, Payne DC, Lopman B, Yen C, Tate JE. Value of post-licensure data on benefits and risks of vaccination to inform vaccine policy: the example of rotavirus vaccines. Vaccine. 2015 Nov 27;33:D55-9

[8]. McGrath EJ, Thomas R, Duggan C, Asmar BI. Pentavalent rotavirus vaccine in infants with surgical gastrointestinal disease. Journal of pediatric gastroenterology and nutrition. $2014 \mathrm{Jul} ; 59(1): 44$

[9]. Cera SM. Intestinal intussusception. Clinics in colon and rectal surgery. 2008 May;21(2):106.

[10]. Mpabalwani EM, Mwenda JM, Tate JE, Parashar UD. Review of naturally occurring intussusception in young children in the WHO African region prior to the era of rotavirus vaccine utilization in the expanded programme of immunization. Journal of tropical pediatrics. 2017 Jun 1;63(3):221-8

[11]. www.medicalnewstoday.com/articles/intussusceptio $\mathrm{n}$ 
[12]. www.learningradiology.com/archives04/COW\%2009 3-intussusception/intussusceptcorrect.htm

[13]. Ordoñez CA, Puyana JC. Management of peritonitis in the critically ill patient.Surgical Clinics. 2006 Dec 1;86(6):1323-49.

[14]. www.sages.org/wiki/intussusception/

[15]. Walter EB, Staat MA. Rotavirus Vaccine and Intussusception Hospitalizations. Pediatrics. 2016 Sep $1 ; 138(3)$.

[16]. Patel MM, López-Collada VR, Bulhões MM, et al. Intussusception risk and health benefits of rotavirus vaccination in Mexico and Brazil. $\mathrm{N}$ Engl $\mathrm{J}$ Med. 2011;364(24):2283-2292. doi:10.1056/NEJMoa1012952

[17]. Yen C, Shih SM, Tate JE, Wu FT, Huang YC, Parashar UD, Hsiung CA. Intussusception-related Hospitalizations among Infants before and after Private Market Licensure of Rotavirus Vaccines in Taiwan, 2001-2013. The Pediatric infectious disease journal. 2017 Oct;36(10):e252

[18]. Cho H-K, Hwang SH, Nam HN, Han K, Kim B, Kong I, et al. (2020) Incidence of intussusception before and after the introduction of rotavirus vaccine in Korea. PLoS ONE 15(8): e0238185. https://doi.org/10.1371/journal.pone.0238185

[19]. Burnett E, Tate JE, Kirkwood CD, Nelson EA, Santosham M, Steele AD, Parashar UD. Estimated impact of rotavirus vaccine on hospitalizations and deaths from rotavirus diarrhea among children $<5$ in Asia. Expert review of vaccines. 2018 May 4;17(5):453-60.

[20]. www.worldsdg2030.org/images/White-paper-Book.pd f.

[21]. Muhsen K, Kassem E, Efraim S, Goren S, Cohen D, Ephros M. Incidence and risk factors for intussusception among children in northern Israel from 1992 to 2009: a retrospective study. BMC pediatrics. 2014 Dec 1;14(1):218.

[22]. Koch J, Harder T, von Kries R, Wichmann O. Risk of Intussusception After Rotavirus Vaccination. Dtsch Arztebl Int. 2017 Apr 14;114(15):255-262. doi: 10.3238/arztebl.2017.0255. PMID: 28468712; PMCID: PMC5424085

[23]. www.who.int/vaccine_safety/committee/topics/vaccin e_safety_blueprint/Dec_2011/en/

[24]. World Health Organization. Global rotavirus information and surveillance bulletin. Geneva: WHO. 2013

[25]. Julie Bines. WHO Post-marketing surveillance of rotavirus vaccine safety [Internet]. WHO. Availablefrom :www.who.int/immunization/document s/WHO_IVB_09.01/en/ [cited 2018 Jun 17]

[26]. Groome MJ, Fairlie L, Morrison J, Fix A, Koen A, Masenya M, Jose L, Madhi SA, Page N, McNeal M, Dally L. Safety and immunogenicity of a parenteral trivalent P2-VP8 subunit rotavirus vaccine: a multisite, randomised, double-blind, placebo-controlled trial. The Lancet Infectious Diseases. 2020 Apr 3 